
\title{
25 Research Suare \\ Case Report: A Rare Case of Brain Metastases in an Elderly Patient with Primary Pancreatic Cancer
}

\section{Sharad Rajpal}

Boulder Neurosurgical Associates

\section{Hash Brown Taha}

University of Colorado Boulder

\section{Lukas Kvascevicius}

Vilnius University: Vilniaus Universitetas

\section{Sigita Burneikiene ( $\nabla$ sigitab@bnasurg.com )}

Justin Parker Neurological Institute https://orcid.org/0000-0002-6508-4140

\section{Research Article}

Keywords: Case report, Brain metastasis, Pancreatic adenocarcinoma, Gross-tumor resection, Stereotactic radiosurgery.

Posted Date: February 16th, 2022

DOI: https://doi.org/10.21203/rs.3.rs-1083132/v1

License: (c) (1) This work is licensed under a Creative Commons Attribution 4.0 International License. Read Full License

Version of Record: A version of this preprint was published at Cureus on August 1st, 2022. See the published version at https://doi.org/10.7759/cureus.27578. 


\section{Abstract}

Pancreatic adenocarcinoma is an extremely aggressive cancer with a low survival rate. Common sites for metastases include the liver and lungs while brain metastases are considered extremely rare, especially in elderly patients. We present an elderly female patient who developed brain metastases 51-months after the initial diagnosis of pancreatic cancer and was treated with gross tumor resection, chemotherapy, and stereotactic radiosurgery. The treatment completely resolved her neurological symptoms but did not result in improved survival for this patient. The patient developed generalized tonic-clonic seizures and was diagnosed with leptomeningeal carcinomatosis and died 5.5 months after tumor resection.

\section{Introduction}

Pancreatic adenocarcinoma (PAC) is an aggressive cancer with an overall 5-year relative survival rate of 9\% [1] and 7.6 per 100,000 incidence rate occurring in North America [2]. The most common sites for metastases include liver, peritoneum, lungs, and bone [3]. Although an actual incidence of brain metastases from pancreatic cancer is unknown, they are considered extremely rare $(0.1-0.6 \%)[4,5]$ with an even lower incidence of antemortem cases reported in the literature $[6,7]$. This number is expected to increase due to improved treatments and prolonged survival. With only 28 cases of antemortem brain metastasis from pancreatic cancer reported in the literature to date [6], we report another rare case in an elderly female patient.

\section{Case Description}

An 80-year-old female patient presented to the emergency department with worsening right upper extremity (RUE) and lower extremity (RLE) weakness, difficulty with fine motor tasks, but intact comprehension and speech. Neurological exam revealed intact sensation, but weakness in the RUE and RLE: $4 / 5$ right grip, $3 / 5$ biceps/triceps, $1 / 5$ right deltoid, $3 / 5$ dorsi- and plantar flexors and $2 / 5$ in hip flexion. The patient had known stage IIB (T3, N1, M0) PAC with liver metastasis, which was diagnosed in 2016. A Whipple procedure was performed at that time, followed by adjuvant chemotherapy and radiotherapy (Fig. 1) with excellent response. Brain computed tomography and magnetic resonance imaging (MRI) studies revealed a right $(18 \times 18 \mathrm{~mm})$ and left $(31 \times 30 \mathrm{~mm})$ frontal lesions with peripheral enhancement and central necrosis with mild adjacent brain edema without midline shift, but slight mass effect on the motor strip (Fig. 2a).

\section{Operation}

The patient was admitted to the hospital and underwent staging which revealed no additional primary or metastatic lesions. The patient was taken to the operating room and underwent bifrontal craniotomy with gross total resection of both tumors. Postoperative MRI scan (Fig. 2b) showed gross total resections of both lesions without residual enhancement. Pathologic analysis of the specimens demonstrated malignant cells consistent with metastatic adenocarcinoma of pancreatic primary exhibiting moderately 
to well-differentiated glandular structures with associated mucin and calcification. Immunostaining was positive for cytokeratin 7, epithelial membrane antigen, CDX2 (patchy) and CK20 (focal) without expression of thyroid transcription factor -1 .

\section{Postoperative Course}

Three weeks postoperatively, the patient had complete resolution of her right-sided weakness and no other neurological deficits.

The patient underwent postoperative stereotactic radiosurgery (SRS) to both tumor bed cavities. The total target dose of 2,700 cGy was prescribed, 900 cGy in 3 fractions. Unfortunately, the patient developed generalized tonic-clonic seizures, was diagnosed with leptomeningeal carcinomatosis, and died 5.5 months after tumor resection.

\section{Discussion}

This case presents an elderly female patient with metastatic adenocarcinoma of pancreatic primary. While advanced age is a risk factor for developing PAC with cases primarily occurring in elderly patients, a median age of 70 [8], brain metastases from PAC most often occur in younger individuals with a median age of 58 years and are often identified postmortem [6]. The median time between the PAC diagnosis and brain metastasis development ranges between 14 and 29 months [6, 9]. The longest time was reported by Lemke et al., [10] the authors presented a 48-year-old female patient who was diagnosed with a right cerebellum metastasis 65-months after the diagnosis of PAC, which was successfully resected followed by radiotherapy with no local or distant recurrences at the 17-year follow-up. In our case, the patient's primary cancer was successfully managed for 51 months until the development of brain metastases.

The optimal treatment strategies for PAC patients with brain metastases are still debatable, but surgical resection is generally recommended, especially for cystic lesions that are larger than $3 \mathrm{~cm}$ and located in eloquent areas [11]. Given the tumor sizes, symptomatology and remission of the primary tumor, surgical resection and adjuvant SRS was considered as a feasible treatment strategy for this patient. The median survival time was 11 months, and the 1 -year local control rate was $73 \%$ in a study that evaluated tumor bed SRS after resection of brain metastases [12]. The authors noted that a lower maximum dose and SRS delay (> 3 weeks) were associated with increased local recurrence. In our case, the patient survived 58 months after the initial diagnosis. Although a full resolution of her neurological symptoms was achieved following gross total resection and adjuvant SRS, it did not result in an overall survival benefit. According to a literature review of the 28 reported antemortem cases so far [6], the survival from diagnosis of brain metastases ranges from 2 weeks to more than 10 years for surgical treatment and from 3 days to more than 1 year for treatments that included best supportive care, chemotherapy and radiation therapy.

\section{Conclusion}


We report an 80-year-old female patient treated with a gross total resection and adjuvant radiosurgery for two bilateral frontal area metastases from pancreatic cancer, which completely resolved her neurological symptoms, but did not result in improved survival for this patient.

\section{Abbreviations}

CGy - centi-gray; MRI - magnetic resonance imaging; PAC - pancreatic adenocarcinoma; RLE - right lower extremity; RUE - right upper extremity; SRS - stereotactic radiosurgery.

\section{Declarations}

Funding: No funding was received

Conflicts of interest/Competing interests: The authors declare that they have no conflict of interest.

Availability of data and material: available

Code availability: not applicable

Authors' contributions: Performing surgery - Sharad Rajpal; Literature search and the first draft - Hash Brown Taha, Lukas Kvascevicius, Revisions and final manuscript - Sigita Burneikiene and Sharad Rajpal. All authors approved the final manuscript.

Ethics approval: Exempt human subject research

Consent to participate: not required

Consent for publication: consented

\section{References}

1. Siegel RL, Miller KD, Jemal A: Cancer statistics, 2020. CA Cancer J Clin. 2020, 70:7-30. $10.3322 /$ caac. 21590

2. Rawla P, Sunkara T, Gaduputi V: Epidemiology of Pancreatic Cancer: Global Trends, Etiology and Risk Factors. World J Oncol. 2019, 10:10-27. 10.14740/wjon1166

3. Yachida S, lacobuzio-Donahue CA: The pathology and genetics of metastatic pancreatic cancer. Arch Pathol Lab Med. 2009, 133:413-422. 10.1043/1543-2165-133.3.413 10.5858/133.3.413

4. Oweira H, Petrausch U, Helbling D, Schmidt J, Mannhart M, Mehrabi A, Schob O, Giryes A, Decker M, Abdel-Rahman O: Prognostic value of site-specific metastases in pancreatic adenocarcinoma: A 
Surveillance Epidemiology and End Results database analysis. World J Gastroenterol. 2017, 23:1872-1880. 10.3748/wjg.v23.i10.1872

5. Cannistra M, Ruggiero M, Zullo A, Serafini S, Grande R, Nardo B: Metastases of pancreatic adenocarcinoma: A systematic review of literature and a new functional concept. Int J Surg. 2015, 21 Suppl 1:S15-21. 10.1016/j.ijsu.2015.04.093

6. Oka Y, Takano S, Kouchi Y, Furukawa K, Takayashiki T, Kuboki S, Suzuki D, Sakai N, Kagawa S, Hosokawa I, Mishima T, Konishi T, Kishimoto T, Ohtsuka M: Simultaneous brain and lung metastases of pancreatic ductal adenocarcinoma after curative pancreatectomy: a case report and literature review. BMC Gastroenterol. 2021, 21:9. 10.1186/s12876-020-01587-3

7. El Kamar FG, Jindal K, Grossbard ML, Mizrachi HH, Kozuch PS: Pancreatic carcinoma with brain metastases: case report and literature review. Dig Liver Dis. 2004, 36:355-360.

10.1016/j.dld.2003.10.019

8. Wang H, Liu J, Xia G, Lei S, Huang X, Huang X: Survival of pancreatic cancer patients is negatively correlated with age at diagnosis: a population-based retrospective study. Sci Rep. 2020, 10:7048. 10.1038/s41598-020-64068-3

9. Kumar A, Dagar M, Herman J, lacobuzio-Donahue C, Laheru D: CNS involvement in pancreatic adenocarcinoma: a report of eight cases from the Johns Hopkins Hospital and review of literature. J Gastrointest Cancer. 2015, 46:5-8. 10.1007/s12029-014-9667-y

10. Lemke J, Barth TF, Juchems M, Kapapa T, Henne-Bruns D, Kornmann M: Long-term survival following resection of brain metastases from pancreatic cancer. Anticancer Res. 2011, 31:4599-4603.

11. Soffietti R, Abacioglu U, Baumert B, Combs SE, Kinhult S, Kros JM, Marosi C, Metellus P, Radbruch A, Villa Freixa SS, Brada M, Carapella CM, Preusser M, Le Rhun E, Ruda R, Tonn JC, Weber DC, Weller M: Diagnosis and treatment of brain metastases from solid tumors: guidelines from the European Association of Neuro-Oncology (EANO). Neuro Oncol. 2017, 19:162-174. 10.1093/neuonc/now241

12. Iorio-Morin C, Masson-Cote L, Ezahr Y, Blanchard J, Ebacher A, Mathieu D: Early Gamma Knife stereotactic radiosurgery to the tumor bed of resected brain metastasis for improved local control. $\mathrm{J}$ Neurosurg. 2014, 121 Suppl:69-74. 10.3171/2014.7.GKS141488

\section{Figures}




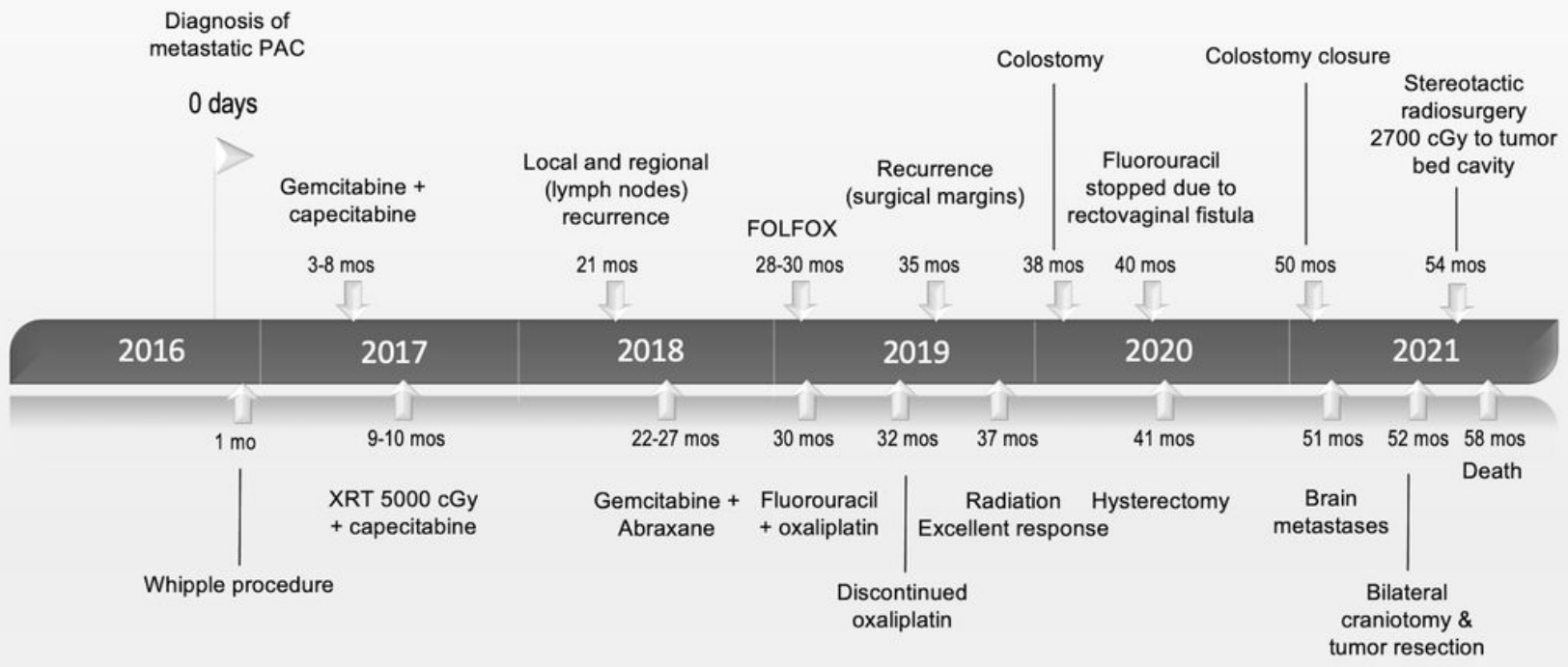

Figure 1

Timeline of patient's treatment course 

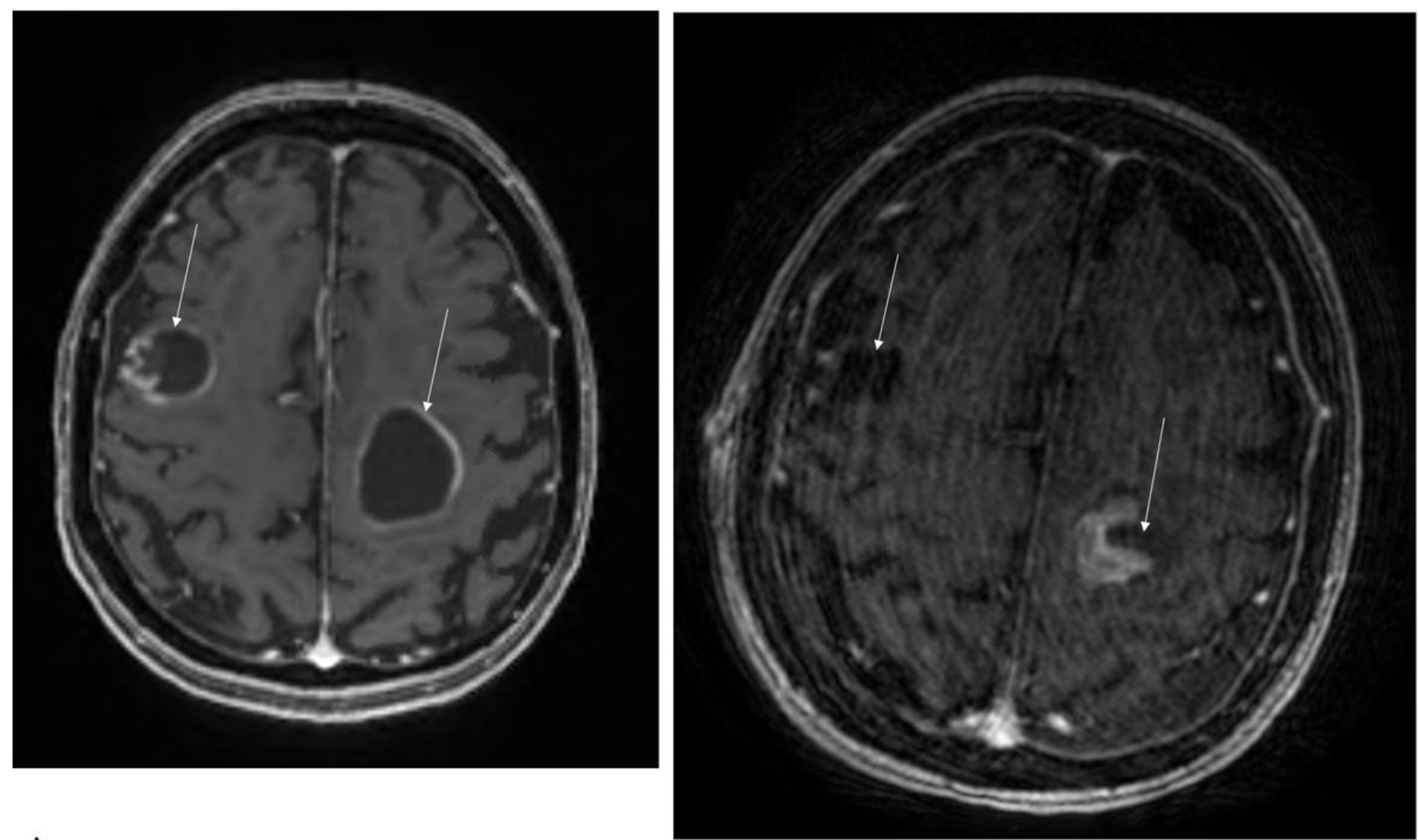

A

B

Figure 2

a: T1-weighted axial MRI view of the brain with contrast, pre-operative scan shows 31 x $30 \mathrm{~mm}$ cystic lesion in the high left frontoparietal junction with rim enhancement inferiorly and $18 \times 18 \mathrm{~mm}$ cystic lesion in the high frontal region with peripheral enhancement ring along the lateral border. b: Motion degraded post-operative image demonstrates interval resection of the left frontoparietal and right posterior frontal lesions with small amount of blood involving the surgical resections (left > right) without evidence of enhancement and a small left posterior convexity subdural hematoma

\section{Supplementary Files}

This is a list of supplementary files associated with this preprint. Click to download.

- CAREchecklistEnglish2013.pdf 